\title{
Uso pesado de álcool por estudantes dos ensinos fundamental e médio de escolas centrais e periféricas de Campinas (SP): prevalência e fatores associados Heavy alcohol use among elementary and high-school students in downtown and outskirts of Campinas City - São Paulo: prevalence and related factors
}

Meire Soldera, ${ }^{a}$ Paulo Dalgalarrondo, ${ }^{a}$ Heleno Rodrigues Corrêa Filho ${ }^{b}$ e Cleide Aparecida M Silvac

Versão original aceita em português
aDepartamento de Psicologia Médica e Psiquiatria - Faculdade de Ciências Médicas da Universidade Estadual de Campinas (FCM / UNICAMP)

${ }^{b}$ Departamento de Medicina Preventiva e Social - Faculdade de Ciências Médicas da Universidade Estadual de Campinas (FCM / UNICAMP)

'Estatística da Comissão de Pesquisa - Faculdade de Ciências Médicas da Universidade Estadual de Campinas (FCM / UNICAMP)

\begin{abstract}
Resumo
0 conhecimento de fatores associados ao uso de álcool na adolescência é de grande relevância, pois permite intervenções visando reduzir comportamentos de risco e o possível início de um uso de álcool progressivamente deletério.

objetivos: Determinar a prevalência do uso pesado de álcool e verificar se variáveis sociodemográficas, culturais e psicopatológicas podem estar influenciando este uso por estudantes dos ensinos fundamental e médio de escolas públicas e particulares da cidade de Campinas (SP).

Métodos: Trata-se de um estudo transversal com uma técnica de amostragem do tipo intencional. Foi utilizado um questionário anônimo, de autopreenchimento, baseado no questionário do Centro Brasileiro de Informações sobre Drogas Psicotrópicas (CEBRID). A amostra foi constituída por 2.287 estudantes dos ensinos fundamental e médio de escolas públicas e particulares da cidade de Campinas (SP), no ano de 1998. Considerou-se uso pesado, de acordo com a World Health Organization (WHO) (1981), o uso de álcool em 20 dias ou mais nos 30 dias que antecederam a pesquisa. Para identificar os fatores que influenciam o uso de drogas, utilizou-se a "Análise de Regressão Logística Politômica - Modelo de Logitos Generalizados". Resultados: 0 uso pesado de álcool foi de 11,9\%, tendo sido maior nos estudantes da escola pública central, do período vespertino, que trabalhavam, de nível socioeconômico $A$ e $B$, que se sentiam pouco apoiados e compreendidos pela família e que apresentavam maior defasagem escolar. Conclusões: Os dados deste estudo indicam que fatores como disponibilidade de dinheiro, trabalho, situações pessoais e familiares desfavoráveis e mau desempenho escolar estão associados ao uso pesado de álcool em estudantes.
\end{abstract}

Descritores: Alcoolismo; Estudantes; Estudos transversais; Inquéritos de morbidade

\begin{abstract}
It is important to identify factors related to heavy alcohol use among adolescents, as this allows interventions aimed at reducing risk behavior and possible increasing harmful use of alcohol.

objectives: To determine the prevalence of heavy alcohol use and investigate the influence of sociodemographic, cultural and psychopathological variables on alcohol use among elementary and high-school students of public and private schools in Campinas, Brazil.

Methods: This is a cross-sectional study using an intentional sampling technique. The questionnaire used was based on the CEBRID (Brazilian Information Center on Psychotropic Drugs) questionnaire and filled out anonymously by the subjects. The sample consisted of 2,287 elementary and high-school students from public and private schools in Campinas, Brazil, during the year of 1998. According World Health Organization criteria (WHO, 1981), alcohol use for 20 days or more in the 30 days prior to the study was considered heavy alcohol use. The "Polytomic Logistic Regression Generalized Logits Model" was applied to identify the factors that influenced heavy alcohol use.

Results: Heavy alcohol use was seen in $11.9 \%$ of the sample, being more frequent among students at the inner-city public school belonging to socioeconomic classes $A$ and $B$, who held down jobs and attended school in the afternoons. These students felt that their families did not understand or support them and also showed lower academic performance at school.

Conclusions: The present study indicates that factors such as availability of funds, work, delayed schooling performance and unfavorable personal and family situations were related to heavy alcohol use among adolescents.
\end{abstract}

Keywords: Alcohoolism; Students; Cross-sectional studies; Morbidity surveys 


\section{Introdução}

0 uso de bebidas alcoólicas inicia-se cedo na vida, entre o início e meio da adolescência, com o grupo de amigos ou mesmo em casa. $^{1-2}$ Este uso implica conseqüências médicas, psicológicas e sociais, podendo ser, para muitos indivíduos, o início da trajetória que conduz à dependência do álcool. ${ }^{2}$ Sendo assim, o conhecimento de fatores associados ao uso de álcool na adolescência é de grande relevância, pois permite intervenções no sentido de reduzir comportamentos de risco.

No Brasil, para a população global (adolescentes, adultos e idosos) a estimativa de prevalência de dependência do álcool varia de 7,6\%, em São Paulo, a 9,2\% em Porto Alegre. 30 álcool é responsável por cerca de $90 \%$ das internações hospitalares por dependência e aparece em $70 \%$ dos laudos cadavéricos por mortes violentas. ${ }^{4}$

Desde 1997, o Centro Brasileiro de Informações sobre Drogas Psicotrópicas (CEBRID) vem realizando levantamentos sobre o uso de drogas por estudantes brasileiros dos ensinos fundamental e médio, em dez capitais brasileiras. Esses levantamentos têm sido muito importantes, pois, além de metodologia adequada, abrangem uma amostra muito grande (15.503 estudantes). 0 uso pesado de álcool (20 vezes ou mais por mês) nesses levantamentos $(1987,1989,1993$ e 1997) foi significativo (de $3,3 \%$ a $10,1 \%$ ). Além disso, os levantamentos indicaram que o uso pesado de álcool aumentou em 8 das 10 capitais estudadas no período de 1987 a 1997.2

0 quarto estudo (1997) mostrou que o uso pesado de álcool foi maior nos estudantes do gênero masculino $(5,2 \%)$ em relação ao feminino $(4,8 \%)$; na classe social $A(10,7 \%)$ em relação à $B(9,1 \%)$, C $(7,6 \%)$, D (6,8\%) e $E(4,9 \%)$; e nos que tinham defasagem escolar - entre os estudantes que faziam uso pesado de álcool, 96,1\% apresentavam defasagem escolar. 0s fermentados foram as bebidas mais consumidas e os bares foram os locais preferidos entre os que fizeram uso pesado. Este estudo revelou também que $68,4 \%$ dos pais bebem muito, contra $6,2 \%$ das mães. ${ }^{2}$

Vários estudos internacionais também mostraram a associação entre uso pesado de álcool por estudantes e variáveis demográficas e psicossociais. Assim, o uso pesado de álcool mostrou-se associado ao nível socioeconômico mais alto, ${ }^{5}$ à defasagem esco$\operatorname{lar}^{6-7}$ e ao beber do pai. ${ }^{8}$

0 objetivo deste trabalho foi determinar a prevalência do uso pesado de álcool por estudantes dos ensinos fundamental e médio de escolas públicas centrais (duas escolas) e periféricas (duas escolas) e particulares (três escolas) da cidade de Campinas (SP). Além disso, visou-se determinar fatores sociodemográficos, culturais e psicopatológicos associados a este uso.

\section{Métodos}

Trata-se de um estudo transversal com uma técnica de amostragem do tipo intencional. Visou-se, com isso, representar 0 contraste entre estudantes de escolas centrais e periféricas, privadas e públicas da cidade, no nível de escolaridade considerado. As linhas gerais desenvolvidas nesta pesquisa seguem, com as adaptações necessárias em função dos objetivos e dimensão do trabalho, o método proposto pelo CEBRID - $1993 .{ }^{9}$

Foi utilizado um questionário anônimo, de autopreenchimento, baseado no questionário do CEBRID. A aplicação foi coletiva, em sala de aula, sem a presença do professor. A opção por este méto- do se deu pelo fato de ser um processo que garante 0 anonimato da informação prestada - o que é essencial quando se trata de assunto de natureza privada ou ilegal, como é o caso das drogas, aumentando, dessa forma, a chance de se obter informações válidas. A utilização do mesmo método permite, também, uma padronização dos estudos e a comparação dos resultados obtidos. Foi realizado, inicialmente, um estudo piloto, quando foram aplicados 607 questionários sobre 0 uso de drogas junto a alunos do ensino médio de duas escolas públicas, utilizando o mesmo questionário utilizado nesta pesquisa.

A partir do estudo piloto, calculou-se o tamanho da amostra no EPI_NF0. Esse cálculo amostral foi feito estimando-se 5\% do erro tipo I (alfa), com população finita estimada em 100.000 alunos para a cidade, no grau sob estudo, sem reposição; prevalência de usuários de 1\%; erros de desenho de no máximo 1\%, com precisão delta de $1 \%$.

Uma vez que se teve como objetivo comparar diferença substancial de comportamento entre escolas públicas e particulares considerando-se as localizações periférica e central para cada nível de escolaridade, tornou-se necessária amostra mínima de 367 estudantes dos ensinos fundamental e médio, em cada um dos três estratos pretendidos, a saber: escolas públicas periféricas, centrais e particulares. Foram compostos, então, seis estratos de 400 alunos, totalizando 2.400 estudantes, distribuídos nas séries de $6^{\underline{a}}$ a $8^{\underline{a}}$ do ensino fundamental e $1^{\underline{a}}$ a $3^{\underline{a}}$ do ensino médio.

A partir das listagens de escolas fornecida pelas Delegacias de Ensino foi feita a escolha intencional das escolas. Determinou-se duas escolas públicas centrais, duas públicas periféricas e três particulares que possuíam ensinos fundamental e médio. A partir das listagens de alunos e turmas de 35 alunos, foram sorteadas seis classes de cada grau por escola. Todos os alunos presentes foram convidados a preencher os questionários e notou-se a presença normal dos alunos durante o inquérito, sem registros de irregularidade na freqüência dos alunos às salas visitadas. Foram incluídos todos os respondentes e excluídos os maiores de 26 anos de idade.

Foram aplicados 2.375 questionários. Não houve nenhuma recusa em respondê-los. Dois questionários foram desprezados por preenchimento incompleto ou não compreensão das perguntas e 86 foram desprezados pois os indivíduos tinham mais de 26 anos, limite etário máximo do estudo.

Portanto, a amostra foi constituída por 2.287 estudantes dos ensinos fundamental e médio de escolas públicas centrais, escolas públicas periféricas e escolas particulares da cidade de Campinas (SP), no ano de 1998. As estimativas oficiais do total de estudantes matriculados na cidade, na época, apontavam cerca de 56 mil estudantes matriculados da $6^{\underline{a}}$ a $8^{a}$ série do ensino fundamental e 52 mil matriculados no ensino médio. Assim, nossa amostra contém, aproximadamente, $2 \%$ do total de estudantes.

Considerou-se uso pesado ${ }^{10} 0$ uso de álcool em 20 dias ou mais nos 30 dias que antecederam a pesquisa. Para identificar os fatores que influenciam o uso de álcool, utilizou-se a "Análise de Regressão Logística Politômica - Modelo de Logitos Generalizados". Este modelo foi escolhido pela propriedade de ajustar múltiplos determinantes atendendo a uma ordem de hierarquia tomada dos referenciais clínicos e das significâncias encontradas nas análises multivariadas. Essa técnica é aplicada quando temos uma variável resposta nominal com mais de dois 
níveis e sem uma ordem inerente, ou quando o modelo de 0 dds Proporcionais é rejeitado. Um Logito é formado pela probabilidade de cada categoria sucessiva sobre a última categoria da resposta. ${ }^{11}$ As variáveis independentes foram: gênero; escola; grau escolar; período em que estudava; anos de defasagem escolar; nível socioeconômico; religião; trabalho; tipo de lazer; situação dos pais; com quem mora; apoio e compreensão familiar; por quem foi criado nos últimos dois anos; apoio e compreensão de amigos(as) e/ou namorado(a); educação religiosa na infância; escore do GHQ12 - General Health Questionnaire 12 (que avalia sintomas psiquiátricos menores, como ansiedade, depressão e insônia - validado no Brasil por Mari \& Willians, 1985).12

A escolha de variáveis significantes foi feita em análises bivariadas prévias. A resposta politômica considerou que o uso de álcool tem gradientes, não cabendo o nível de resposta sim ou não. A ênfase dada ao uso pesado não pode excluir os níveis intermediários, implicando que uma análise dicotômica seria enganosa.

A organização das variáveis independentes procurou explicar apenas relações de ocorrência sem a elaboração implícita de um modelo causal que também estaria prejudicado pelo tipo de estudo transversal. Excluímos confundimentos previamente (sexo e idade e suas associações com outras variáveis estudadas, tais como defasagem escolar e trabalho), através da verificação de que estes fatores estavam fortemente correlacionados com outros fatores de risco que permaneceram no modelo. Não foram estudadas interações, tendo em vista a busca de efeitos principais. 0 nível socioeconômico permaneceu no modelo mesmo tendo sido suposto como confundimento, uma vez que não perdeu significância após os ajustes na prestação do tipo de escola (pública central, pública periférica e particular).

\section{Resultados}

\section{Aspectos gerais da amostra}

Foram estudados 2.287 estudantes. Destes, 1.188 (52,0\%) eram do sexo masculino e 1.096 (48,0\%) do sexo feminino. A média de idade foi de 15,8 \pm 2,7 (mínima de 11 anos e máxima de 26); 781 (34,1\%) nas escolas públicas centrais, $763(33,6 \%)$ nas escolas públicas periféricas e 738 (32,3\%) estudavam nas escolas particulares; $1.159(50,8 \%)$ cursavam o ensino fundamental e $1.122(49,2 \%)$ cursavam o ensino médio.

\section{Prevalência do uso pesado de álcool}

Considerando-se o uso pesado (uso de 20 vezes ou mais no mês), nesta amostra de estudantes o álcool foi utilizado por 269 jovens $(11,9 \%)$. Entre os rapazes, $186(15,9 \%)$, e entre as garotas, 83 $(7,7 \%)$; entre os estudantes das escolas públicas centrais, 114 $(14,8 \%)$ usavam álcool pesadamente, das escolas públicas periféricas, 65 (8,6\%), e das escolas particulares, 90 (12,3\%).

A média de idade da primeira experimentação foi de $12,4 \pm 2,9$ (mínima de dois anos e máxima de 21 anos).

\section{Análise multivariada}

A idade foi testada como variável contínua e não houve significância para permanecer no modelo. 0s confundimentos testados com a variável idade excluíram esta variável por ser colinear com outras variáveis sociais que permaneceram. Foram igualmente testados, sem significância no modelo multivariado, os efeitos da escolaridade, tanto por grau como por período de estudo (matutino, vespertino e noturno).

Sexo e idade foram consideradas variáveis confundidoras e excluídas da análise para dar chance ao aparecimento das demais variáveis estudadas diante do fenômeno da colinearidade. Mais interessante que apontar a exposição de meninos mais velhos ao uso de drogas é verificar que o trabalho é aquele que tem possibilidade de ser considerado um marcador social. 0 trabalho é passível de observação e ação social e não a progressão da idade ou pertencer ao sexo masculino, que além de representarem lugar comum na interpretação do fenômeno, têm implicação óbvia sobre a condição social de acesso ao consumo. Existe, de fato, a associação entre sexo, idade e trabalho. A retirada do fator sexo e idade representa o destaque ao fator trabalho.

A chance do uso pesado de álcool no estudante que trabalhava foi 2,2 vezes maior que a do que não trabalhava; nos que se sentiam muito pouco apoiados e compreendidos pela família, 1,9 $(1 / 0,533)$ vezes maior que nos que se sentiam muito apoiados e compreendidos; do período matutino, $1,8(1 / 0,528)$ vezes maior que do período noturno; do período vespertino 1,62 vezes maior que do período matutino; das classes $A$ e $B, 1,7$ vezes maior que a classe C; da escola particular, $1,5(1 / 0,658)$ vezes maior que da escola pública periférica; da escola pública central, 1,5 vezes maior que da escola particular. Cada ano de defasagem escolar aumentou em 1,16 vezes a chance do estudante fazer uso pesado de álcool (Tabela 1).

\section{Discussão}

A taxa de prevalência de uso pesado de álcool encontrada no presente estudo é claramente mais elevada do que as encontradas nos levantamentos nas 10 capitais brasileiras, realizados pelo CEBRID (11,9\% neste estudo, contra valores que ficam em torno de $7,0 \%$ nas capitais). Como nossos dados foram colhidos em 1998 (e os levantamentos do CEBRID são referentes à 1987, 1989, 1993 e 1997), isto pode estar refletindo o aumento de uso pesado de álcool em estudantes brasileiros, aumento este verificado pelas pesquisas do próprio CEBRID. ${ }^{2}$

Esta alta freqüência de uso pesado de álcool pode também estar indicando um uso maior em cidade interiorana de médio porte, numa região brasileira relativamente rica, em comparação às capitais estudadas.

0 álcool geralmente emerge como a droga mais comum usada por adolescentes nas pesquisas nacionais e internacionais.4,13-21 Além disso, é importante salientar que um estudo mostrou que 0 álcool, assim como a maconha, foi uma das substâncias que apresentaram maior associação com comportamento sexual de risco mais freqüente. 22

Estudos mostraram que o uso de álcool, de modo geral, começa na infância e consolida-se na adolescência. ${ }^{23-24} 0$ s resultados do presente trabalho mostraram que a média de idade da primeira experimentação do álcool se deu muito cedo (12 anos). Isto é preocupante, pois quanto mais cedo inicia-se o contato com o álcool, maior é a probabilidade de que se estabeleça a dependência desta droga. 25

Em relação às variáveis sociodemográficas pesquisadas, o "trabalho" foi uma das variáveis mais importantes; os estudantes que trabalhavam usavam significativamente mais álcool de forma pesada. Outros estudos já mostraram de forma consistente um 
Tabela 1 - Estimativas da regressão logística politômica multivariada - modelo generalizado para a intensidade do uso de álcool comparada ao não uso

\begin{tabular}{|c|c|c|c|c|c|c|c|c|c|}
\hline & \multicolumn{3}{|c|}{ Uso pesado } & \multicolumn{3}{|c|}{ Uso freqüente } & \multicolumn{3}{|c|}{ Uso no mês } \\
\hline & p-valor & or* $^{*}$ & $I C^{\star \star}(95 \%)$ & p-valor & or & IC (95\%) & p-valor & or & IC (95\%) \\
\hline \multicolumn{10}{|l|}{ Tipo de escola } \\
\hline Central & 0,0001 & 1,515 & $(0,968 ; 2,370)$ & 0,1752 & 0,737 & $(0,464 ; 0,569)$ & 0,0000 & 1,651 & $(1,200 ; 2,272)$ \\
\hline \multicolumn{10}{|l|}{$\begin{array}{l}\text { Nivel } \\
\text { socioeconômico }\end{array}$} \\
\hline$A+B$ & 0,0172 & 1,689 & $(1,144 ; 2,491)$ & 0,0032 & 1,954 & $(1,270 ; 3,004)$ & 0,0008 & 1,422 & $(1,099 ; 1,839)$ \\
\hline \multicolumn{10}{|l|}{$\begin{array}{l}\text { Período de } \\
\text { estudo }\end{array}$} \\
\hline Tarde & 0,0008 & 1,620 & $(0,972 ; 2,702)$ & 0,0582 & 2,011 & $(1,156 ; 3,497)$ & 0,2633 & 1,103 & $(0,777 ; 1,566)$ \\
\hline Noite & 0,0012 & 0,528 & $(0,294 ; 0,950)$ & 0,8638 & 1,491 & $(0,801 ; 2,774)$ & 0,2764 & 0,847 & $(0,548 ; 1,308)$ \\
\hline $\begin{array}{l}\text { Trabalho } \\
\text { (trabalhava) }\end{array}$ & 0,0000 & 2,215 & $(1,526 ; 3,210)$ & 0,0006 & 2,014 & $(1,348 ; 3,008)$ & 0,5825 & 1,079 & $(0,823 ; 1,414)$ \\
\hline \multicolumn{10}{|l|}{ Lazer } \\
\hline Esportes & 0,3994 & 1,283 & $(0,831 ; 1,980)$ & 0,0036 & 1,690 & $(1,051 ; 2,718)$ & 0,0002 & 1,583 & $(1,169 ; 2,143)$ \\
\hline $\begin{array}{l}\text { Não tinha lazer + } \\
\text { lazer familiar }\end{array}$ & 0,7750 & 1,111 & $(0,645 ; 1,913)$ & 0,0048 & 0,604 & $(0,300 ; 1,222)$ & 0,0038 & 0,828 & $(0,556 ; 1,231)$ \\
\hline $\begin{array}{l}\text { Sentia-se } \\
\text { parcialmente } \\
\text { apoiado }\end{array}$ & 0,2216 & 0,572 & $(0,333 ; 0,982)$ & 0,4852 & 0,444 & $(0,265 ; 0,744)$ & 0,1906 & 0,929 & $(0,625 ; 1,380)$ \\
\hline $\begin{array}{l}\text { Defasagem } \\
\text { escolar }\end{array}$ & 0,0000 & 1,155 & $(1,100 ; 1,214)$ & 0,0016 & 1,092 & $(1,034 ; 1,154)$ & 0,0000 & 1,099 & $(1,060 ; 1,139)$ \\
\hline
\end{tabular}

maior uso de drogas entre estudantes que trabalham. . $3,26-28^{3}$

Pode-se hipotetizar que a associação entre uso pesado de álcool e trabalho dá-se por diferentes fatores: 0 estresse conseqüente de ter que assumir precocemente uma função laboral, a disponibilidade financeira decorrente de estar trabalhando ou padrões de socialização vinculados ao mundo do trabalho.

0 resultado do presente estudo, no que se refere ao trabalho, nos leva a refletir sobre crenças bem estabelecidas na sociedade brasileira, como a de que o tempo livre é um fator de risco para 0 uso de drogas. Esta concepção implica na identificação de um estereótipo: a do estudante pobre, que passa seu tempo livre na rua, sendo um sujeito potencialmente inclinado ao uso de drogas. ${ }^{27}$ Para Carvalho \& Carlini-Cotrim, ${ }^{27}$ a discussão referente ao trabalho dos adolescentes deve-se deslocar do simplório "fazer" versus "não fazer" para a do "como fazer". Assim, ela defende que um jovem realizado em suas potencialidades, e não apenas ocupado, deva ser a meta adequada de programas que visem promover a saúde mental do adolescente e inibir o uso perigoso de álcool.

No presente estudo, encontrou-se que o uso pesado de álcool, além de associar-se ao trabalho, esteve associado a pertencer às classes sociais A e B. Estes dados corroboram outros estudos que mostram a relação entre o consumo de álcool e maior nível socioeconômico ${ }^{2,5}$ e trabalho.

Uma possível interpretação seria a de que a disponibilidade financeira poderia representar um fator de risco significativo, pois estes estudantes teriam maior facilidade de adquirir 0 álcool e freqüentar locais de consumo, como bares, festas e boates.

Em relação às diferentes escolas pesquisadas (particulares e públicas centrais e periféricas), pode-se identificar que, curiosamente, as escolas públicas centrais aproximam-se mais das particulares do que das públicas periféricas. Nas públicas centrais e particulares verificou-se um uso pesado em 14,8\% e 12,3\%, respectivamente. Já nas escolas públicas periféricas notou-se um menor uso pesado de álcool (8,6\%). Esses resultados podem estar indicando que os estudantes das escolas periféricas, por disporem de menos dinheiro, consomem menos o álcool. Pode-se se aventar a hipótese também de que possuir menos dinheiro pode ser apenas um marcador de uma situação de desfavorecimento social e não a causa do menor consumo de álcool. Além disso, é possível que estudantes mais comprometidos com álcool e drogas, nesses bair- 
ros pobres periféricos, já tenham espirrado do sistema escolar. 0 período do dia de freqüência à escola (manhã, tarde ou noite) que se mostrou mais significativo foi o período da tarde. Este dado é de difícil interpretação, entretanto, pode-se supor que estudantes do período da tarde saiam da escola e dirijam-se com mais freqüência a locais para beber do que estudantes dos outros períodos.

Neste estudo, também verificou-se a importância do ambiente e estrutura familiar como possível fator protetor para o uso pesado de álcool. Identificou-se o uso menor em estudantes que, de certa forma, se sentiam apoiados e compreendidos pela família.

Algumas pesquisas revelaram um uso de drogas com risco para jovens pertencentes a famílias com pais separados ou famílias com relacionamentos muito deteriorados. ${ }^{29-33} \mathrm{Um}$ estudo mostra que o clima do ambiente familiar é mais importante que o estatuto conjugal dos pais. Dessa forma, nas famílias sem violência, nas quais os problemas são conversados, pais vivem juntos e se preocupam com os filhos, haveria menor probabilidade de um uso abusivo de álcool e drogas. ${ }^{30}$

Chama muito a atenção também o resultado encontrado nesta pesquisa relacionando maior uso pesado de álcool à defasagem escolar. Outras pesquisas já identificaram associação entre uso de drogas e menor rendimento escolar.2,6-7,20,34 Pode-se aventar aqui algumas hipóteses: a primeira é de que alterações neuropsicológicas decorrentes do uso de drogas estariam dificultando a aprendizagem escolar; a segunda, de que o atraso escolar revelaria algum tipo de desajustamento social, problemas de conduta ou dificuldade de disciplina. Outra hipótese seria a de que o uso pesado de álcool interferiria no rendimento escolar. Finalmente, podese aventar que o baixo rendimento escolar pode afetar a auto-estima e induzir ao uso de álcool. Estas várias hipóteses não são, em tese, excludentes.

\section{Conclusão}

0 presente estudo indicou que o uso pesado de álcool, estando associado à maior disponibilidade financeira (classe social, trabalho e escolas públicas centrais e particulares), padrões de socialização mais "adultomórficos" (trabalho), pior relacionamento familiar (sentir-se menos apoiado e compreendido) e pior desempenho escolar, talvez esteja a nos revelar que a relação entre sofrimento psicossocial e maior facilidade de acesso ao álcool sejam dimensões-chave na compreensão do envolvimento de estudantes com o álcool.

Trabalho desenvolvido no Departamento de Psicologia Médica e Psiquiatria FCM/UNICAMP.

Artigo baseado na Tese de Doutorado "Uso de drogas por estudantes de $1^{0}$ e $2^{\circ}$ graus na cidade de Campinas-SP: prevalência e fatores sociodemográficos, culturais e psicopatológicos associados", apresentada no ano de 2001, na Universidade Estadual de Campinas (UNICAMP).

Financiamento: Fundação de Amparo à pesquisa do Estado de São Paulo FAPESP.

Recebido em 29.07.2002

Aceito em 14.11.2003

\section{Referências}

1. Laranjeira R, Nicastri S. Abuso e dependência de álcool e drogas. In: Almeida 0, Dractu L, Laranjeira R. Manual de psiquiatria. Rio de Janeiro: Guanabara Koogan; 1996. p. 83-112.

2. Galduróz JCF, Noto AR. Uso pesado de álcool entre estudantes de $1^{\circ}$ e $2^{\circ}$ graus da rede pública de ensino em dez capitais brasileiras. J Bras Dep Quím. 2000;1(1):25-32.

3. Almeida-Filho N, Mari JJ, Coutinho E, França JF, Fernandes JG, Andreoli $S B$, et al. Estudo multicêntrico de morbidade psiquiátrica em áreas urbanas brasileiras (Brasília, São Paulo, Porto Alegre). Rev ABP-APAL. 1992;14(3):93-104.

4. Galduróz JCF, Noto AR, Carlini EA. Tendências do uso de drogas no Brasil: sintese dos resultados obtidos sobre o uso de drogas entre estudantes do $1^{\circ}$ e $2^{\circ}$ graus em 10 capitais brasileiras (1987 - 1989 - 1993 - 1997). São Paulo: Centro Brasileiro de Informações Sobre Drogas Psicotrópicas: Departamento de Psicobiologia da Escola Paulista de Medicina; 1997.

5. Pechansky F. 0 uso de bebidas alcoólicas em adolescentes residentes na cidade de Porto Alegre. Características de consumo e problemas associados [tese]. Rio Grande do Sul: Universidade Federal do Rio Grande do Sul; 1993.

6. Bachman SJ, Johnston LD, O'Malley PM. Smoking, drinking, and drug use among American high school students: correlates and trends, 1977-1979. Am J Public Health. 1995;171(1):59-69.

7. Johnson V, Bennett ME. Assessing and traking family histories of alcoholism. J Stud Alcohol. 1995;56(6):654-60.

8. Jennison KM, Johnson KA. Alcohol dependence in adult children of alcoholics: longitudinal evidence of early risk. J Drug Educ. 1998;28(1):19-37. 9. Carlini-Cotrim B, Barbosa MTS. Pesquisas epidemiológicas sobre o uso de drogas entre estudantes: um manual de orientações gerais. São Paulo: Centro Brasileiro de Informações Sobre Drogas Psicotrópicas Departamento de Psicobiologia da Escola Paulista de Medicina; 1993.

10. Edwards G. Arif A. Nomenclature and classification of drug and alcohol-related problems: a WHO, Memorandum. Bull World Health Organ. 1981;59(2):225-45

11. Stokes ME, Davis CS, Koch GG. Categorical Data Analysis - Using the SAS System; 1995.

12. Mari JU, Williams P. A comparison of the validy of two psychiatric screening questionnaires (GHQ-12 and SRQ-20) in Brazil, using relative operating characteristic (ROC) analysis. Psychol Med. 1985;15(3):651-9. 13. Sarinana MEC, Maya MA, Aquilar MA. Consumo de substancias tóxicas y tabaco entre la poblacion estudantil de 14 - 18 años. Salud Publica Méx.1982;24(5):565-74.

14. Swadi H. Drug and substance use among 3.333 London adolescents. $\mathrm{Br}$ J Addict. 1988;83:935-42

15. Gilvarry E, McCarthy, McArdle P. Substance use among schoolchildren in the north of England. Drug Alcohol Depend. 1995;37(3):255-9.

16. Stevens $M$, Youells $F$, Whaley F, Linsey $S$. Drug use prevalence in a rural school-age population: the New Hampshire survey. Am J Prev Med. 1995; 11(2):105-13.

17. Kandel DB, Davies M. High School Students who use crack and other drugs. Arch Gen Psychiatry, 1996;53(1):71-80.

18. Khan N, Arnott R. Substance use among rural secondary schools in Zimbabwe: patterns and prevalence. Cent Afr J Med. 1996;42(8):223-9.

19. Kuria MW. Drug abuse among urban as compared to rural secondary schools students in Kenya: a short comunication. East Afr Med $\mathrm{J}$. 1996;73(5):339.

20. Muza GM, Betiol H, Mucillo G, Barbieri MA. Consumo de substâncias psicoativas por adolescentes escolares de Ribeirão Preto, SP (Brasil). I Prevalência do consumo por sexo, idade e tipo de substância. Rev Saúde Pública. 1997;31(1):21-9.

21. Deitos FT, Santos RP, Pasqyalotto AC, Segat FM, Guillande S, Benvegnú LA. Prevalência do consumo de tabaco, álcool e drogas ilícitas em estudantes de uma cidade de médio porte no sul do Brasil Inform Psiquiatr. 1998;17(1):11-6.

22. Scivoletto S, Tsuji RK, Abdo CHN, Queiroz S, Andrade AG, Gattaz WF. Relação entre consumo de drogas e comportamento sexual de estudantes de $2^{\circ}$ grau de São Paulo. Rev Bras Psiquiatr. 1999;21(2):87-94.

23. Berjano RM, Foguet JMB, Garcia MS, Gonzales AMC. The consumption of tobacco, alcohol and other drugs by adolescent Spanish students. Gac Sanit. 1998;12(6):263-71. 
24. Alamendras MF, Ripoll RM, Dijk A, Garcia AD, Sanmarti LS. Alcohol consumption among high school students in Barcelona, Spain. J Stud Alcohol. 1999;60(2):228-33.

25. Williams CL, Perry CL. Preventing alcohol problems during adolescence. Lessons from project Northland. Alcohol Youth 1998;22(2). [cited 2004 Aug 2]. Available from; http://www.niaaa.nih.gov/publications/arh222/107-116.pdf

26. Barbosa MTS, Carlini-Cotrim B, Silva-Filho AR. 0 uso de tabaco por estudantes de primeiro e segundo graus em dez capitais brasileiras: possiveis contribuições da estatística multivariada para a compreensão do fenômeno. Rev Saúde Pública. 1989;23(5):401-9.

27. Carvalho V, Carlini-Cotrim B. Atividades extra-curriculares e prevenção ao abuso de drogas: uma questão polêmica. Rev Saúde Pública. 1992;26(3):145-9.

28. Valois RF, Dunham ACA, Jackson KL, Waller J. Association Between Employment and Substance abuse behaviors among public high school adolescents. J Adolesc Health. 1999;25(4):256-63.

29. Costa IRA. Os melhores amigos - o uso de drogas e a visão de mundo de jovens de camadas médias urbanas [dissertação]. Rio de Janeiro (RJ): Pontifícia Universidade Católica do Rio de Janeiro; 1993.

30. Carvalho V, Pinsky I, Souza e Silva R, Carlini-Cotrim B. Drug and alcohol use and family characteristics: a study among Brazilian high-school students. Addiction. 1995;90(1):65-72.

31. Carlini-Cotrim B, Carlini EA. 0 consumo de solventes e outras drogas em crianças e adolescentes de baixa renda na Grande São Paulo. Parte I: Estudantes de $1^{\circ}$ e $2^{\circ}$ graus da Rede Estadual. Rev ABP-APAL. 1987;2(9):4958.

32. Hoffmann JP. Os efeitos da estrutura familiar e relações familiares no adolescente usuário de maconha. Int J Addict. 1995;30(10):1207-41.

33. Miller P. Family Structure, Personality, Drinking, Smoking And Illicit Drug Use: A Study of UK Teenagers. Drug Alcohol Depend.e 1997;45(12):121-9.

34. Mcc Miller P, Plant M. Drinking, smoking, and illicit drug use among 15 and 16 year olds in the United Kingdom. BMJ.1996;313(7054):394-7. Comment in: BMJ. 1996;313(7054):375.

Correspondência

Meire Soldera

Rua Pascoal Notte, 32 - Parque Taquaral 13087-380 Campinas, SP, Brasil

Tel.: (19) 9795-1361 